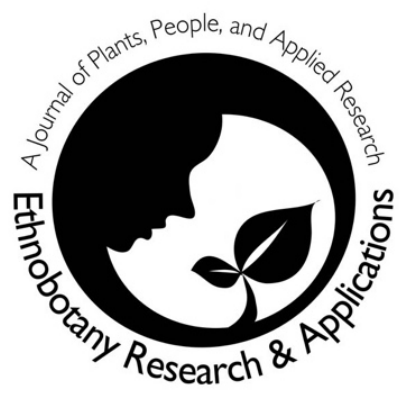

\title{
Putative Anthelmintic Plants Used in Traditional Medicine System of Kokrajhar District, India
}

Ananta Swargiary*, Manita Daimari and Mritunjoy Kumar Roy

Correspondence

Ananta Swargiary*, Manita Daimari and Mritunjoy Kumar Roy

Department of Zoology, Pharmacology and Bioinformatics Lab., Bodoland University, Kokrajhar, 783370, Assam, India

*Corresponding Author: ananbuzoo101@gmail.com

Ethnobotany Research \& Applications 22:10 (2021)

\section{Research}

\begin{abstract}
Background:Traditional medicine (TM) is a common practice among tribal communities of India. The present study has been designed to collect the ethnomedicinal plants used by the Bodo community of Kokrajhar district of India traditionally consumed to cure helminth infection.
\end{abstract}

Methods: A total of 54 villages were surveyed from the Kokrajhar district. Twenty adjacent villages were taken as a cluster, and one informant was interviewed face-to-face with the help of a readymade questionnaire. Traditional knowledge system of anthelmintic herbal medicines such as the name of the plants, parts used, methods of formulation, and mode of uses was collected. The demography of the informants was also collected.

Results: Total of 64 species of traditionally used anthelmintic plants belonging to 38 families were documented from Kokrajhar district, India. Andrographis paniculata was the most popular plant, followed by Alstonia scholaris, Ananas comosus, and Azadirachta indica. Poaceae was the most popular plant family with six species of anthelmintic plants. The leaves were the most commonly used plant-part (63\%), followed by barks and tubers. Raw, decoction and infusion were the standard method of traditional formulations reported from the district. Fresh and raw plant parts in the form of paste or balls and oral consumption were the primary means of administration. Demographically, most of the knowledge bearers were found to be illiterate and aged above 50 years of age.

Conclusions: The medicinal plants reported in the present study could be a source of important medicines. A proper scientific study needs to be carried out to study the efficacy of the traditional formulations to ascertain their bioactivity.

Keywords: Ethnomedicine, Anthelmintic, Tribal Community, Kokrajhar

\section{Background}

The traditional use of plants and animals as medicines to cure diseases is an age-old practice throughout the world. Ethnomedicinal knowledge systems are transferred from one generation to the next without any formal means of communication (Samy \& Ignacimuthu 2000; Verpoorte et al. 2005; Vitalini et al. 2013). Despite better healthcare facilities of the contemporary world, there is growing attention towards plant-derived compounds and their biological properties. According to the WHO Traditional Medicinal Strategy 2014-23 (WHO 2013), there is a rising 
demand for ethnomedicinal practices and practitioners worldwide. Plants are readily available, possesses fewer side effects, and are sometimes more efficacious than commercial drugs. Because of these properties, the application of plant-based products and medicines are increasing day by day (WHO 2013, Barkaoui et al. 2017; Aziz et al. 2018). Over the decades, scientific studies have explored the bioactivity of several plants, leading to the development of new therapeutic drugs. Many studies have revealed medicinal properties against various diseases, including helminthiasis (Maphosa \& Masika 2010; Barkaoui et al. 2017; Aziz et al. 2018; Chaudhury et al. 2015; Adeniyi et al. 2018). In the run for new drugs discovery, the ethnomedicinal knowledge system can play a significant role. Today, the pharmacological study based on ethnomedicinal knowledge system is a major research area in the drug discovery pipeline. Ethnomedicinal studies have gained interest among the researchers to find important plant species and develop new medicines against the diseases (Pendry et al. 2005; Watkins et al. 2011). In many societies, the practice of herbal medicine systems is popular and culturally accepted by the people. For example, in India, people with different cultural backgrounds practice traditional medicines for common diseases (Gogtay et al. 2002). Helminthiasis is a severe tropical disease that affects millions of people and the livestock as well as the poultry industries (Kumar \& Nain 2013; Das \& Laha 2017; Dixit et al. 2017; Salam \& Azam 2017). According to WHO, about 870 million children living in tropical countries worldwide are affected by helminthiasis. India alone contributes nearly $25 \%$ of it, with 220 million children that require medical attention (Lobo et al. 2011). Despite improved health facilities, sanitation, drinking water, and mass deworming programs, helminthiasis remain a grave health issue in several states of India (Salam \& Azad 2017). In addition to poor economic conditions, most rural Indians cannot access commercial anthelmintic drugs because of geographical isolation and the high cost of the drugs. However, as an alternative, locally available traditional medicines become the first choice for healthcare needs (Tandon et al. 2011).

The northeastern region (NER) of India, consisting of eight member states, including Assam, covers an area of $262,230 \mathrm{~km}^{2}$. NER is one of the $35^{\text {th }}$ biodiversity hotspots on earth with rich flora and fauna (Mittermeier et al. 2011). As per the 2011 census of India, NER has over 220 ethnic groups speaking more than 220 different languages. In Assam, tribal communities are concentrated mainly in the Bodoland Territorial Region (BTR), formed by curving eight districts of Assam (BTC 2003). There are four districts under Bodoland Region, namely Kokrajhar, Chirang, Baksa, and Udalguri, administered by the Bodoland Territorial Council (BTC). Bodo, Rabha, and Rajbonghis represent the major tribal communities of the Bodoland area. The practice of the traditional medicine systems is widespread and prominent among the tribal communities of BTR. However, the practice of ethnomedicine differs from community to community. Different communities have different ways and means of practising and preserving their ethnomedicinal knowledge systems (Kotoky \& Das 2008; Shankar et al. 2012). It varies from common household remedies to the treatment of severe diseases with specialised treatment processes. People that are involved in traditional healing are locally known as 'kaviraja' or 'ojhas'. Several studies have investigated and documented the ethnobotanical aspects of herbal medicines against several diseases in Assam (Purkayastha et al. 2005; Das et al. 2006; Narzary et al. 2013; Swargiary et al. 2013, 2016; Tamuli \& Ghosal 2017; Sonowal \& Barua 2017). Like many other parts of Assam, the Bodoland area is full of natural flora and fauna. Despite abundant medicinal plants, no scientific study has been conducted to explore the ethnobotanical knowledge of this part of India. Therefore, the present study is designed to explore the diversity of medicinal plants used to cure helminth infection (locally known as 'kirml') by the Bodo community of Kokrajhar district of India.

\section{Methods and Materials}

Study area and its description

The survey was carried out in the Kokrajhar district of Assam (India), which covers 3,169.22 sq. km (longitude $89^{\circ} 46^{\prime}$ East to $90^{\circ} 38^{\prime}$ East and latitude $26^{\circ} 19^{\prime}$ North to $26^{\circ} 54^{\prime}$ North). Located on the northern side of the River Brahmaputra, the district is plain land with small hillocks bounded by the Himalayan Foothills of Bhutan on the northside, Chirang district on the east, West Bengal on the west, and Dhubri district on the southside. With lush green forest, and rich flora and fauna, the climatic conditions of the district are sub-tropical with warm and humid summer $\left(27.64^{\circ} \mathrm{C}\right.$ to $\left.31.67^{\circ} \mathrm{C}\right)$ followed by cool and dry winter $\left(19.34^{\circ} \mathrm{C}\right.$ to $\left.23.66^{\circ} \mathrm{C}\right)$ with an annual average rainfall of $2,400 \mathrm{~mm}$ to $3,000 \mathrm{~mm}$. Out of 887,142 populations inhabiting 1053 villages, nearly $94 \%$ live in rural areas, while $31 \%$ belong to scheduled tribe communities. According to the 2011 census, Kokrajhar has a literacy rate of $65.2 \%$ (Census of India, 2011). For administrative purposes, the Kokrajhar district has been divided into small Community Developmental Blocks (CDB). The entire district consists of 11 CDBs, namely, (1) Kokrajhar (Titaguri), (2) Dotoma, (3) Kochugaon, (4) Gossaigaon, (5) Hatidhura, (6) Bilasipara (Part), (7) Chapar-Salkocha (Part), (8) Rupshi (Part), (9) Mahamaya (Part), (10) Golokganj (Part), and (11) Debitola (Part). Out of the 11 CDBs, Kochugaon is having the highest number of villages (238 nos.) followed by Kokrajhar (224 nos.), Dotoma (172 nos.), Debitola (138 nos.), Gossaigaon (112 nos.), Hatidhura (44 nos.), Rupsi (43 nos.), Chapar-Salkocha (36 nos.), Mahamaya (34 nos.), 
Golokganj (8 nos.) and Bilasipara with four numbers of villages. Kokrajhar is the biggest CDB, followed by Kochugaon, Debitola, Dotoma, Gossaigaon, Rupsi, Chapar-Salkocha, Hatidhura, Mahamaya, Bilasipara, and Golokganj.

\section{Data collection and identification of plants}

The survey and collection of anthelmintic medicinal plants were carried out from April to October 2018. The information regarding the medicinal plants was collected in a CDB-wise manner with the help of local traditional healers. The ethnomedicinal data was also collected from older people having traditional medicine knowledge. Both males and females were involved in the survey work. Within every CDB, approximately 20 nearby villages were taken as a single cluster, and one sample was collected. The information was collected by face-to-face interview manner with the help of a readymade questionnaire. Based on the number of villages of CDBs, the sample size also varied from one CDB to another. The information collected from informants mainly included the informer's biodata, plant(s) part(s) used, traditional formulation processes and mode of administration. Total, 54 informants were interviewed from 54 different villages under the district, and traditional knowledge about anthelmintic plants was collected. Sample plants were collected, herbarium sheets were prepared and submitted to the Department of Botany, Bodoland University, to identify the plant samples.

\section{Data analysis}

All the statistical calculations, graphs were carried out in Microsoft Excel and OriginPro-8.5 software. The data were analysed by comparing several parameters such as the number of plant species, families, plant parts used, modes of utilisation, habit, and habitat.

The quantitative analysis was carried out to evaluate the importance of plants following Hussain et al. (2018).

Frequency of Citation (FC) is the number of informants who mentioned a certain species.

Relative frequency of citation (RFC): It is obtained by dividing FC by total number of informants (N). The value of RFC indicates the citing percentage of each species of plant. RFC is calculated by using the following formula (Tardio et al. 2008):

$$
\mathrm{RFC}=\mathrm{FC} / \mathrm{N}
$$

The value of RFC varies from zero (when nobody cites to a plant as important) to one (when all the informants consider a certain species important).

Family importance value (FIV): FIV indicates the local importance of the families of plant species and is calculated by counting the percentage of informants mentioning a specific family (Vitalini et al. 2013).

$$
\text { FIV }=[F C \text { (Family) } / \mathrm{N}] \times 100
$$

\section{Results}

The present study surveyed 54 villages from 11 CDBs of Kokrajhar district to collect anthelmintic medicinal plants. Traditional healers and older knowledgeable persons were involved in the data collection. The name of CDBs, name of the villages and their geographical locations are shown in Table 1. A highest of 12 villages were surveyed from Kochugaon CDB, followed by Kokrajhar, Dotoma, Debitola, Gossaigaon, Rupsi, Chapar-Salkocha, Hatidhura, and Mahamaya development blocks. No data was collected from Golokganj (Part) and Bilasipara (part) because of fewer villages and the absence of tribal communities in the particular blocks (Table 2). Figure 1 indicates the information collection sites. Out of the total 54 informants, 37 were males, and 17 were females. Most of the knowledge bearers were aged people. About $74 \%$ of the total informants were $>50$ years old, followed by $26 \%$ in $40-50$ years old. In terms of literacy, most of the informants (48\%) had school-level education, $19 \%$ have college-level education, and $33 \%$ do not have any formal education. Among the 54 informants, very few (7\%) have salaried government jobs. Most of the informants from Kokrajhar and Kochugaon CDBs were literate, while informants from other CDBs were illiterate. The study also observed that both the literate and illiterates possess almost equal knowledge in terms of plant citation. On average, one literate informant named 4.6 plant citations, while illiterate named 5.0 plant citations. About $72 \%$ of the total informants were professional healers who practice traditional healing on a daily basis and earn little money. In comparison, $28 \%$ of older knowledgeable people do not get involved in traditional healing practices. 
Table 1. Demographic characteristics of informants of Kokrajhar district, India.

\begin{tabular}{|c|c|c|c|c|c|c|c|c|}
\hline \multirow[b]{2}{*}{ Block } & \multicolumn{3}{|c|}{ Literacy } & \multicolumn{2}{|c|}{ Age group } & \multicolumn{2}{|c|}{ Informants } & \multirow[b]{2}{*}{ Total } \\
\hline & School & College & Illiterate & $>\mathbf{5 0}$ & $40-50$ & Kaviraja & Older & \\
\hline Kokrajhar & 3 & 6 & 2 & 9 & 2 & 8 & 3 & 11 \\
\hline Kochugaon & 7 & 2 & 3 & 9 & 3 & 10 & 2 & 12 \\
\hline Dotoma & 3 & 2 & 4 & 6 & 3 & 4 & 5 & 9 \\
\hline Debitola & 4 & 0 & 4 & 4 & 4 & 7 & 1 & 8 \\
\hline Gossaigaon & 4 & 0 & 3 & 5 & 2 & 5 & 2 & 7 \\
\hline Rupsi & 2 & 0 & 1 & 3 & 0 & 2 & 1 & 3 \\
\hline $\begin{array}{l}\text { Chapar- } \\
\text { Salkocha }\end{array}$ & 2 & 0 & 0 & 2 & 0 & 1 & 1 & 2 \\
\hline Hatidhura & 0 & 0 & 1 & 1 & 0 & 1 & 0 & 1 \\
\hline Mahamaya & 1 & 0 & 0 & 1 & 0 & 1 & 0 & 1 \\
\hline Total & 26 & 10 & 18 & 40 & 14 & 39 & 15 & 54 \\
\hline
\end{tabular}

Table 2. List of villages where information regarding traditionally used anthelmintic medicinal plants was collected along with the geographical location.

\begin{tabular}{|c|c|c|}
\hline CD Block & List of villages & Geographical location \\
\hline \multirow[t]{11}{*}{ Kokrajhar } & Athiabari & 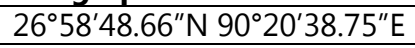 \\
\hline & Bashbari & 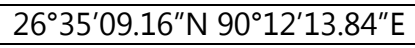 \\
\hline & Batipara & $26^{\circ} 22^{\prime} 40.17^{\prime \prime} \mathrm{N} 90^{\circ} 15^{\prime} 23.14^{\prime \prime} \mathrm{E}$ \\
\hline & Bhowraguri & $26^{\circ} 36^{\prime} 09.86^{\prime \prime} \mathrm{N} 90^{\circ} 14^{\prime} 24.07^{\prime \prime} \mathrm{E}$ \\
\hline & Chilaguri & 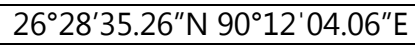 \\
\hline & Latagaon & $26^{\circ} 29^{\prime} 03.41^{\prime \prime} \mathrm{N} 90^{\circ} 20^{\prime} 36.29^{\prime \prime} \mathrm{E}$ \\
\hline & Mawriagaon-II & $26^{\circ} 27^{\prime} 04.83^{\prime \prime} \mathrm{N} 90^{\circ} 08^{\prime} 40.14^{\prime \prime} \mathrm{E}$ \\
\hline & Pakriguri & $26^{\circ} 31^{\prime} 00.58^{\prime \prime} \mathrm{N} 90^{\circ} 14^{\prime} 32.44^{\prime \prime} \mathrm{E}$ \\
\hline & Ranighuli & 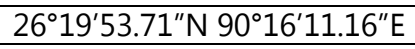 \\
\hline & Shamthaibari & 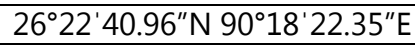 \\
\hline & Sutarpara & 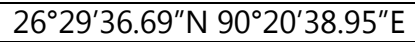 \\
\hline \multirow[t]{2}{*}{ Chapar-Salkocha (Pt.) } & Bamungaon Pt.IV & $26^{\circ} 29^{\prime} 05.81^{\prime \prime} \mathrm{N} 90^{\circ} 28^{\prime} 05.83^{\prime \prime} \mathrm{E}$ \\
\hline & Chakrasila Pt.I & 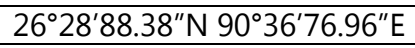 \\
\hline \multirow[t]{9}{*}{ Dotoma } & Baghmar & $26^{\circ} 28^{\prime} 38.78^{\prime \prime} \mathrm{N} 90^{\circ} 08^{\prime} 48.01^{\prime \prime} \mathrm{E}$ \\
\hline & Bauti & 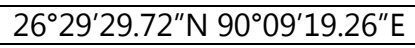 \\
\hline & Bhalukmari & $26^{\circ} 29^{\prime} 56.05^{\prime \prime} \mathrm{N} 90^{\circ} 12^{\prime} 12.12^{\prime \prime} \mathrm{E}$ \\
\hline & Boragari & 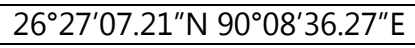 \\
\hline & Dangarkhuti & $26^{\circ} 27^{\prime} 21.23^{\prime \prime} \mathrm{N} 90^{\circ} 12^{\prime} 21.58^{\prime \prime} \mathrm{E}$ \\
\hline & Dotoma Bazar & 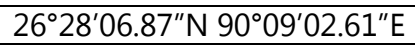 \\
\hline & Ghoramar & $26^{\circ} 32^{\prime} 54.97^{\prime \prime} \mathrm{N} 90^{\circ} 48^{\prime} 20.83^{\prime \prime} \mathrm{E}$ \\
\hline & Narenguri & 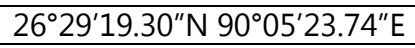 \\
\hline & Dumuriguri & 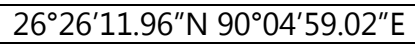 \\
\hline \multirow[t]{7}{*}{ Gossaigaon } & Balapara-Siljhar & $26^{\circ} 32^{\prime} 24.60^{\prime \prime} \mathrm{N} 90^{\circ} 10^{\prime} 40.43^{\prime \prime} \mathrm{E}$ \\
\hline & Banglabari & $26^{\circ} 39^{\prime} 90.55^{\prime \prime} \mathrm{N} 90^{\circ} 03^{\prime} 27.32^{\prime \prime} \mathrm{E}$ \\
\hline & Bhowraguri & $26^{\circ} 36^{\prime} 06.15^{\prime \prime} \mathrm{N} 90^{\circ} 14^{\prime} 24.83^{\prime \prime} \mathrm{E}$ \\
\hline & Gossaigaon-I & $26^{\circ} 42^{\prime} 84.87^{\prime \prime} \mathrm{N} 89^{\circ} 99^{\prime} 88.33^{\prime \prime} \mathrm{E}$ \\
\hline & Madati & 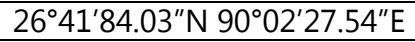 \\
\hline & Mowamari & $26^{\circ} 42^{\prime} 46.90^{\prime \prime} \mathrm{N} 90^{\circ} 10^{\prime} 77.73^{\prime \prime} \mathrm{E}$ \\
\hline & Singimari-II & $26^{\circ} 36^{\prime} 04.09^{\prime \prime} \mathrm{N} 89^{\circ} 99^{\prime} 01.02^{\prime \prime} \mathrm{E}$ \\
\hline \multirow[t]{12}{*}{ Kochugaon } & Balagong & $26^{\circ} 58^{\prime} 17.06^{\prime \prime} \mathrm{N} 90^{\circ} 07^{\prime} 53.78^{\prime \prime} \mathrm{E}$ \\
\hline & Chengmari FV & $26^{\circ} 32^{\prime} 55.30^{\prime \prime} \mathrm{N} 89^{\circ} 56^{\prime} 37.73^{\prime \prime} \mathrm{E}$ \\
\hline & Gombariguri & $26^{\circ} 51^{\prime} 13.51^{\prime \prime} \mathrm{N} 90^{\circ} 11^{\prime} 98.16^{\prime \prime} \mathrm{E}$ \\
\hline & Haldibari FV & $26^{\circ} 36^{\prime} 08.48^{\prime \prime} \mathrm{N} 89^{\circ} 54^{\prime} 08.78^{\prime \prime} \mathrm{E}$ \\
\hline & Jonaligaon FV & $26^{\circ} 64^{\prime} 69.75^{\prime \prime} \mathrm{N} 89^{\circ} 94^{\prime} 36.99^{\prime \prime} \mathrm{E}$ \\
\hline & Karikar FV & 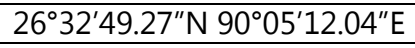 \\
\hline & Kumguri FV & $26^{\circ} 53^{\prime} 98.81^{\prime \prime} \mathrm{N} 90^{\circ} 15^{\prime} 97.63^{\prime \prime} \mathrm{E}$ \\
\hline & Kumtola FV & 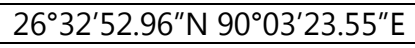 \\
\hline & Lotamari FV-II & 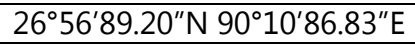 \\
\hline & Salbari FV & $26^{\circ} 56^{\prime} 50.57^{\prime \prime} \mathrm{N} 90^{\circ} 00^{\prime} 08.17^{\prime \prime} \mathrm{E}$ \\
\hline & Takampur FV & $26^{\circ} 60^{\prime} 29.82^{\prime \prime} \mathrm{N} 90^{\circ} 03^{\prime} 51.70^{\prime \prime} \mathrm{E}$ \\
\hline & Kathalguri & $26^{\circ} 52^{\prime} 17.60^{\prime \prime} \mathrm{N} 90^{\circ} 00,58.82^{\prime \prime} \mathrm{E}$ \\
\hline Debitola (Pt.) & Borshijhora Pt-II & $26^{\circ} 21^{\prime} 65.21^{\prime \prime} \mathrm{N} 89^{\circ} 96^{\prime} 23.53^{\prime \prime} \mathrm{E}$ \\
\hline
\end{tabular}




\begin{tabular}{|c|c|c|}
\hline & Daibari Pt.-I & $26^{\circ} 26^{\prime} 92.97^{\prime \prime} \mathrm{N} 89^{\circ} 97^{\prime} 80.08^{\prime \prime} \mathrm{E}$ \\
\hline & Daimaguri-I & $26^{\circ} 30^{\prime} 46.58^{\prime \prime} \mathrm{N} 90^{\circ} 05^{\prime} 36.79^{\prime \prime} \mathrm{E}$ \\
\hline & Dampur -II & $26^{\circ} 12^{\prime} 20.56^{\prime \prime} \mathrm{N} 90^{\circ} 02^{\prime} 53.42^{\prime \prime} \mathrm{E}$ \\
\hline & Debitola Pt.-II & $26^{\circ} 15^{\prime} 76.43^{\prime \prime} \mathrm{N} 90^{\circ} 00^{\prime} 75.26^{\prime \prime} \mathrm{E}$ \\
\hline & Dholagaon Pt.-I & 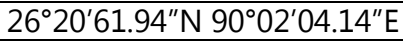 \\
\hline & Duligaon Pt.-III & $26^{\circ} 25^{\prime} 98.01^{\prime \prime} \mathrm{N} 90^{\circ} 07^{\prime} 24.97^{\prime \prime} \mathrm{E}$ \\
\hline & Kazigaon Pt.-I & $26^{\circ} 19^{\prime} 21.79^{\prime \prime} \mathrm{N} 89^{\circ} 99^{\prime} 46.81^{\prime \prime} \mathrm{E}$ \\
\hline Mahamaya (Pt.) & Silbari & $26^{\circ} 13^{\prime} 52.68^{\prime \prime} \mathrm{N} 89^{\circ} 97^{\prime} 82.28^{\prime \prime} \mathrm{E}$ \\
\hline \multirow[t]{3}{*}{ Rupsi (Pt.) } & Basbari & $26^{\circ} 18^{\prime} 93.98^{\prime \prime} \mathrm{N} 89^{\circ} 90^{\prime} 26.52^{\prime \prime} \mathrm{E}$ \\
\hline & Dukhisukhijhar Pt.-I & $26^{\circ} 16^{\prime} 60.90^{\prime \prime} \mathrm{N} 89^{\circ} 89^{\prime} 27.91^{\prime \prime} \mathrm{E}$ \\
\hline & Malatijhora & $26^{\circ} 16^{\prime} 97.28^{\prime \prime} \mathrm{N} 89^{\circ} 95^{\prime} 99.30^{\prime \prime} \mathrm{E}$ \\
\hline Hatidhura (Pt.) & Hatidhura & $26^{\circ} 29^{\prime} 14.89^{\prime \prime} \mathrm{N} 90^{\circ} 15^{\prime} 41.86^{\prime \prime} \mathrm{E}$ \\
\hline
\end{tabular}

*Part (Pt.) means some villages of Debitola, Mahamaya, Rupsi, Chapar-Salkocha, and Hatidhura block comes under the Dhubri district. Some under Kokrajhar district, and the villages that come under Kokrajhar district is written as 'Part'. FV - forest village.

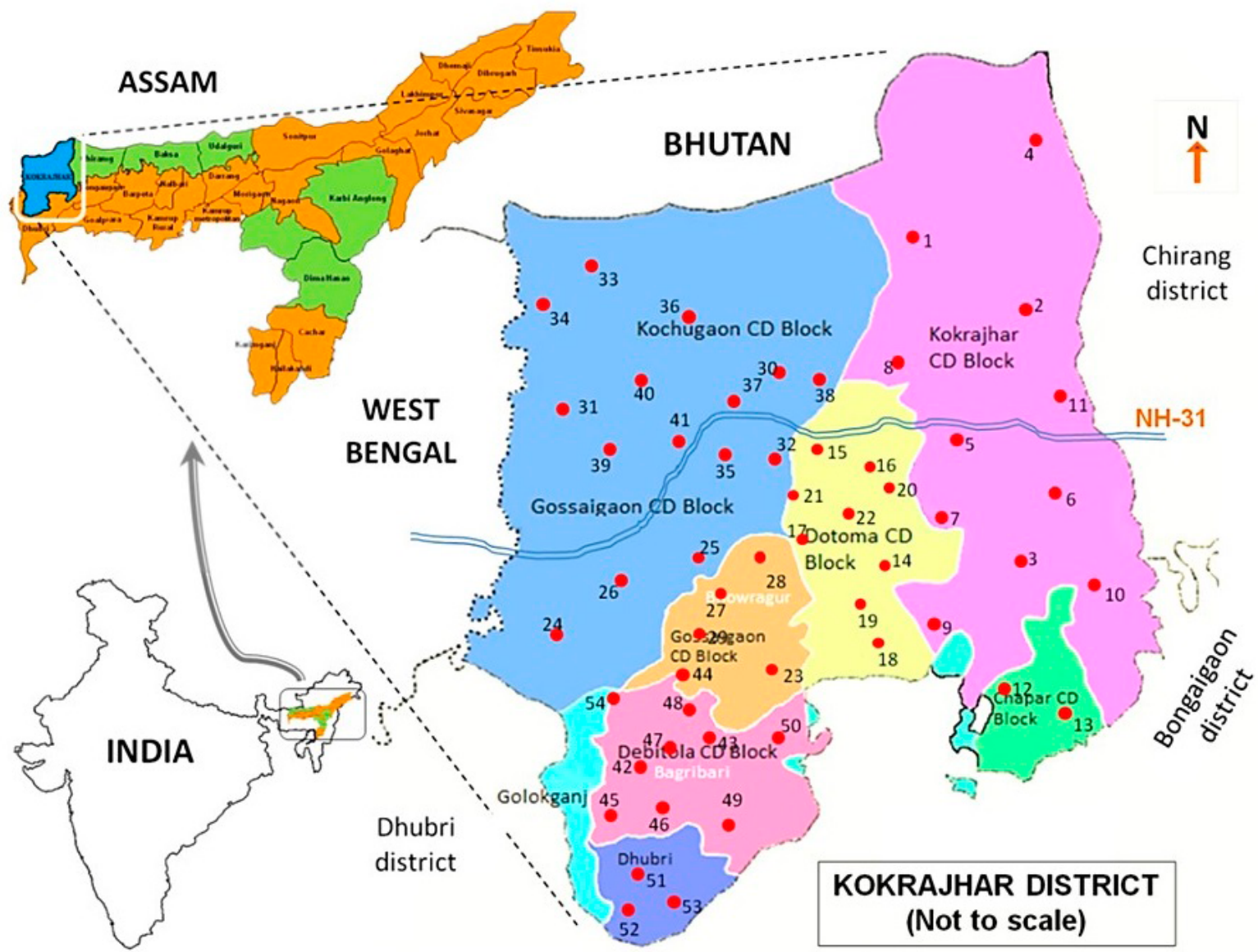

Figure 1. Map of Kokrajhar district and community development blocks indicating the information collection sites. Data collection sites were shown in red spots. The number represents the serial number of Table 1 . NH-31National Highway no. 31.

The name of the plant species (sp.), local name (in Bodo), habit, habitat, parts used, and the traditional formulations is shown in Table 3. A total of 169 plant citations were reported from the survey data. Total of 64 species belonging to 38 families used in the traditional formulations of anthelmintic medicine were documented from the study area. Out of total citations, $23 \mathrm{sp}$. were repeated more than once by the informants consisting of about $75 \%$ (127 citations) of the total citations, while $25 \%$ (42 citations) do not have any repetitions. The FC and and RFC values are presented in Table 3. Plants belonging to 15 families were found more popular with more than one species citation. Six plants were reported from the Poaceae, followed by four sp. from Arecaceae, three sp. from Apocynaceae, Asteraceae, Euphorbiaceae, Fabaceae, Lamiaceae, Rutaceae, and Zingiberaceae. Two plant species were reported from Acanthaceae, Polygonaceae, Rubiaceae, Solanaceae, and Verbenaceae. The percent relative importance of family is shown in Figure 2. 
Table 3 List of plant species, parts used and mode of traditional formulation, and identification number used in the traditional medicine system of tribal communities of Kokrajhar district to control helminth infection.

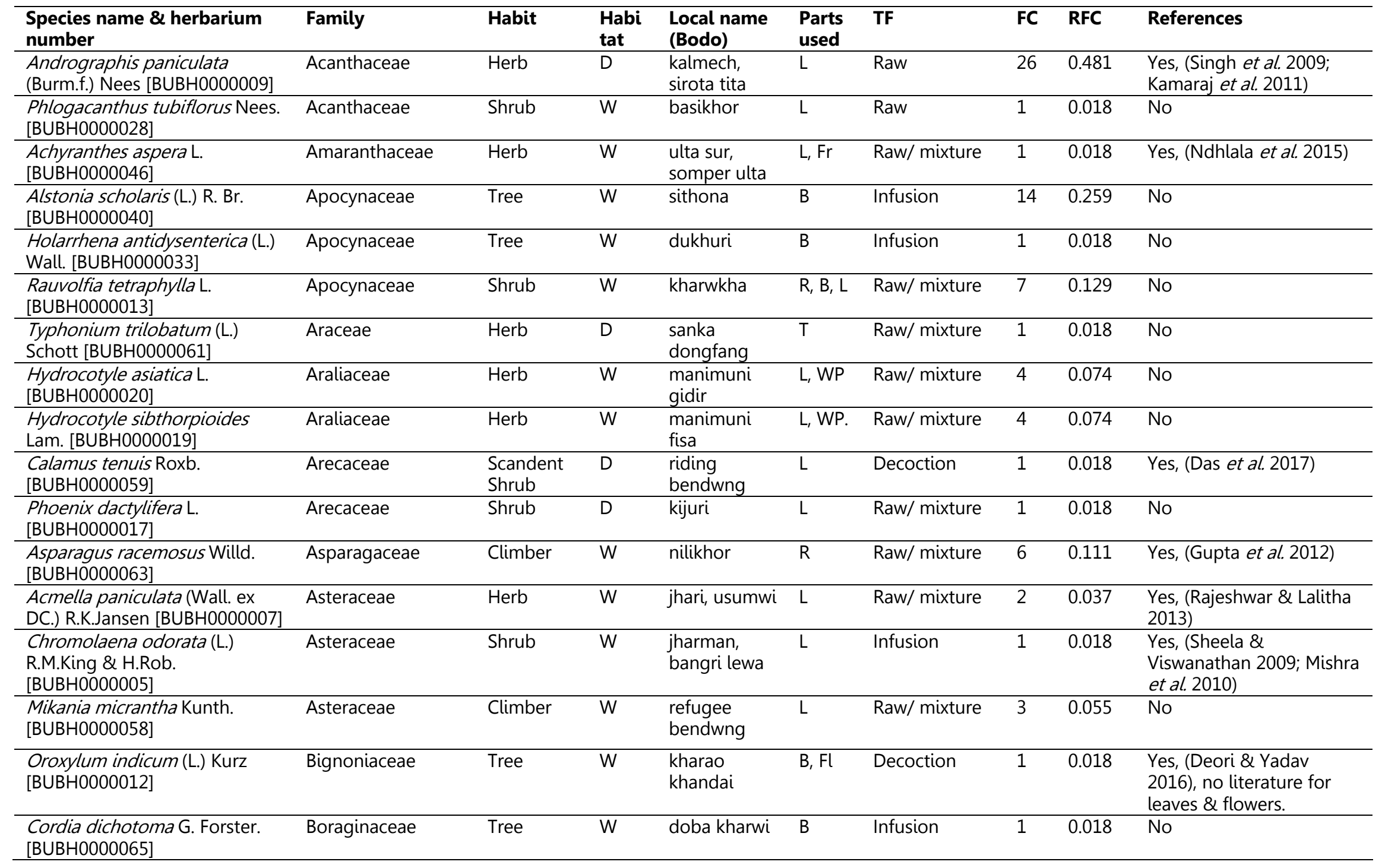


Ethnobotany Research and Applications

\begin{tabular}{|c|c|c|c|c|c|c|c|c|c|}
\hline $\begin{array}{l}\text { Ananas comosus (L.) Merr. } \\
\text { [BUBH0000025] }\end{array}$ & Bromeliaceae & Herb & $\mathrm{D}$ & anaros & $\mathrm{Lt}$ & $\begin{array}{l}\text { Infusion/ } \\
\text { mixture }\end{array}$ & 12 & 0.222 & $\begin{array}{l}\text { Yes, (Hordegen et al. } \\
\text { 2006; Domingues et al. } \\
\text { 2013; Ahmed et al. 2014) }\end{array}$ \\
\hline $\begin{array}{l}\text { Terminalia chebula Retz. } \\
\text { [BUBH0000062] }\end{array}$ & Combretaceae & Tree & $\mathrm{D}$ & silikha & $\mathrm{Fr}$ & Raw/ mixture & 1 & 0.018 & $\begin{array}{l}\text { Yes, (Dwivedi et al. 2008; } \\
\text { Behera \& Bhatnagar } \\
\text { 2018) }\end{array}$ \\
\hline $\begin{array}{l}\text { Bryophyllum pinnatum (Lam.) } \\
\text { Oken [BUBH0000057] }\end{array}$ & Crassulaceae & Herb & $\mathrm{D}$ & paat gaja & $\mathrm{L}$ & Decoction & 1 & 0.018 & Yes, (Lunkad et al. 2016) \\
\hline $\begin{array}{l}\text { Dryopterix filix-mas (L.) Schott } \\
\text { [BUBH0000060] }\end{array}$ & Dryopteridaceae & Herb & $\mathrm{W}$ & $\begin{array}{l}\text { sal } \\
\text { daokhumwi }\end{array}$ & $\mathrm{L}$ & Decoction & 1 & 0.018 & Yes, (Urban et al. 2008) \\
\hline $\begin{array}{l}\text { Euphorbia neriifolia L. } \\
\text { [BUBH0000038] }\end{array}$ & Euphorbiaceae & Shrub & $\mathrm{D}$ & sijou & $\mathrm{L}, \mathrm{Lx}$ & Raw/ mixture & 1 & 0.018 & No \\
\hline $\begin{array}{l}\text { Phyllanthus emblica L. } \\
\text { [BUBH0000023] }\end{array}$ & Euphorbiaceae & Tree & $\mathrm{D}$ & amla & $\mathrm{Fr}$ & Raw & 2 & 0.037 & No \\
\hline $\begin{array}{l}\text { Ricinus communis L. } \\
\text { [BUBH0000003] }\end{array}$ & Euphorbiaceae & Shrub & $\mathrm{D}$ & indi & $\mathrm{L}$ & $\begin{array}{l}\text { Infusion/ } \\
\text { mixture }\end{array}$ & 2 & 0.037 & $\begin{array}{l}\text { Yes, (Zahir et al. 2012; } \\
\text { Mahadev et al. 2017) }\end{array}$ \\
\hline Cassia fistula L. [BUBH0000043] & Fabaceae & Tree & W & sonalu & $\mathrm{L}$ & $\begin{array}{l}\text { Infusion/ } \\
\text { mixture }\end{array}$ & 1 & 0.018 & No \\
\hline $\begin{array}{l}\text { Sesbania grandiflora (L.) Pers. } \\
\text { [BUBH0000004] }\end{array}$ & Fabaceae & Tree & W & jayanti & $\mathrm{L}$ & Raw & 1 & 0.018 & $\begin{array}{l}\text { Yes, (Karumari et al. 2014; } \\
\text { Meenakshisundaram et al. } \\
\text { 2016) }\end{array}$ \\
\hline $\begin{array}{l}\text { Clerodendrum infortunatum } \mathrm{L} \text {. } \\
\text { [BUBH0000047] }\end{array}$ & Lamiaceae & Shrub & W & mwkhwna & $\mathrm{L}$ & Decoction & 1 & 0.018 & $\begin{array}{l}\text { Yes, (Das et al. 2011; } \\
\text { Swargiary et al. 2016) }\end{array}$ \\
\hline $\begin{array}{l}\text { Leucas aspera (Willd.) Link. } \\
\text { [BUBH0000010] }\end{array}$ & Lamiaceae & Herb & $\mathrm{W}$ & kansinsa & $\mathrm{L}$ & Raw & 2 & 0.037 & Yes, (Agarwal et al. 2011) \\
\hline $\begin{array}{l}\text { Ocimum sanctum } \mathrm{L} \text {. } \\
\text { [BUBH0000045] }\end{array}$ & Lamiaceae & Shrub & $\mathrm{D}$ & $\begin{array}{l}\text { tulungsi } \\
\text { (fisa) }\end{array}$ & $\mathrm{L}$ & Raw/ Mixture & 1 & 0.018 & $\begin{array}{l}\text { Yes, (Kalarickal et al. 2015, } \\
\text { Kanojiya et al. 2015) }\end{array}$ \\
\hline $\begin{array}{l}\text { Peliosanthes bakeri Hook.f. } \\
\text { [BUBH0000039] }\end{array}$ & Liliaceae & Herb & $\mathrm{W}$ & sikho & $\mathrm{T}$ & Infusion & 1 & 0.018 & No \\
\hline $\begin{array}{l}\text { Lindernia crustacea (L.) F.Muell. } \\
\text { [BUBH0000048] }\end{array}$ & Linderniaceae & Herb & $\mathrm{W}$ & na bikhi & $\mathrm{L}$ & Decoction & 1 & 0.018 & No \\
\hline $\begin{array}{l}\text { Punica granatum L. } \\
\text { [BUBH0000031] }\end{array}$ & Lythraceae & Shrub & $\mathrm{D}$ & dalim & $\begin{array}{l}\text { B } \\
\text { (root) }\end{array}$ & Raw & 1 & 0.018 & $\begin{array}{l}\text { Yes, (Aggarwal \& Bagai } \\
\text { 2017; Yones et al. 2016) }\end{array}$ \\
\hline $\begin{array}{l}\text { Azadirachta indica A. Juss. } \\
\text { [BUBH0000051] }\end{array}$ & Meliaceae & Tree & $\mathrm{D}$ & neem & $\mathrm{L}$ & $\begin{array}{l}\text { Raw/ mixture/ } \\
\text { Decoction }\end{array}$ & 10 & 0.185 & $\begin{array}{l}\text { Yes, (Costa et al. 2006; } \\
\text { Chagas et al. 2008; Iqbal } \\
\text { et al. 2010) }\end{array}$ \\
\hline
\end{tabular}


Ethnobotany Research and Applications

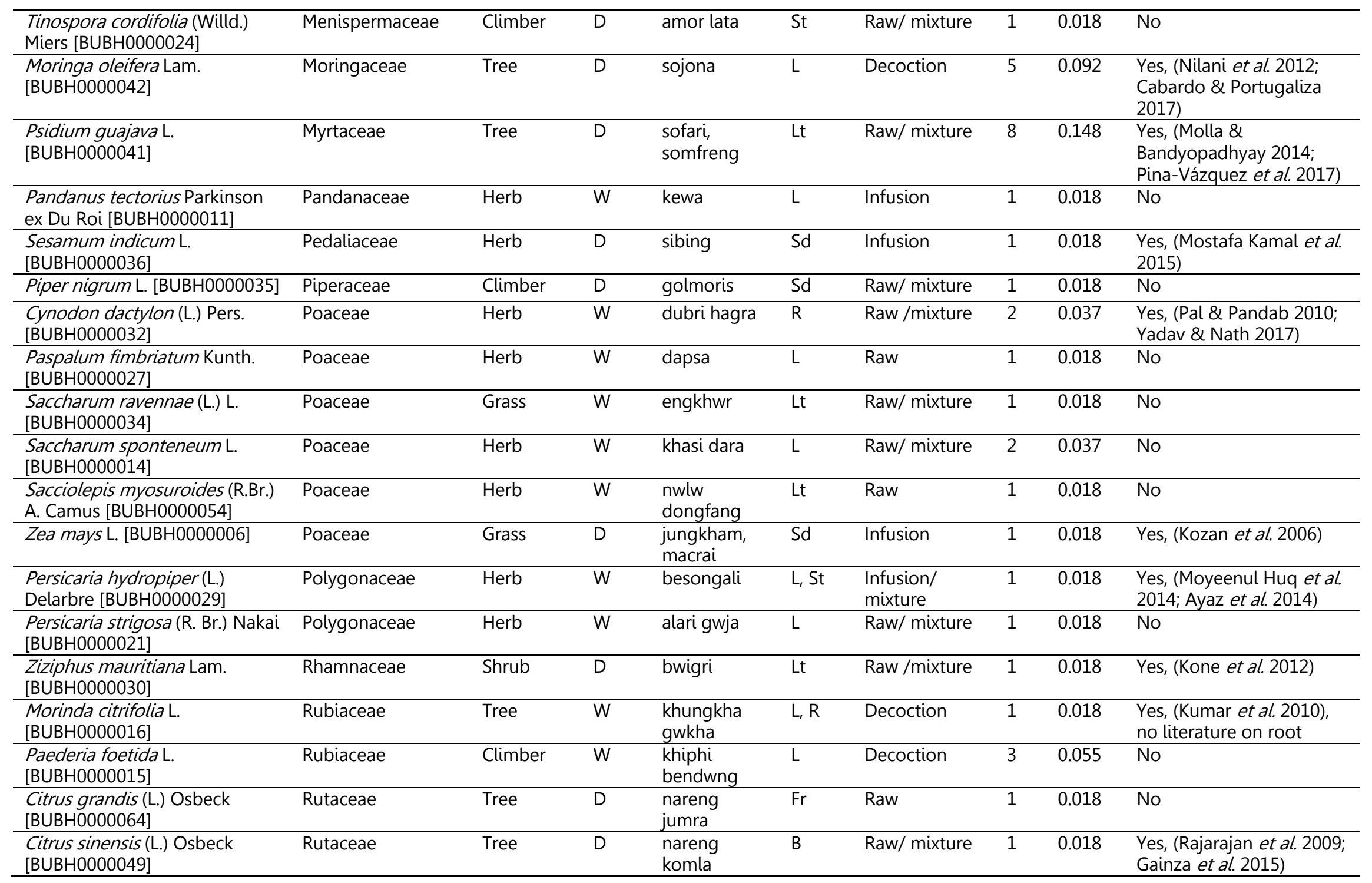


Ethnobotany Research and Applications

\begin{tabular}{|c|c|c|c|c|c|c|c|c|c|}
\hline $\begin{array}{l}\text { Murraya koenigii (L.) Spreng. } \\
\text { [BUBH0000055] }\end{array}$ & Rutaceae & Shrub & W & nwrsing & $\mathrm{L}$ & Raw/ mixture & 1 & 0.018 & $\begin{array}{l}\text { Yes, (Molla \& } \\
\text { Bandyopadhyay 2016; } \\
\text { Swargiary et al. 2016) }\end{array}$ \\
\hline $\begin{array}{l}\text { Cardiospermum halicacabum L. } \\
\text { [BUBH000001] }\end{array}$ & Sapindaceae & $\begin{array}{l}\text { Herb } \\
\text { (Climber) }\end{array}$ & W & alaishree & $\mathrm{L}$ & Raw/ mixture & 1 & 0.018 & No \\
\hline $\begin{array}{l}\text { Capsicum frutescens } \mathrm{L} . \\
{[\mathrm{BUBH} 0000026]}\end{array}$ & Solanaceae & Herb & $\mathrm{D}$ & $\begin{array}{l}\text { banlow } \\
\text { bwrdwn }\end{array}$ & Sd & Raw/ Mixture & 1 & 0.018 & Yes, (Vinayaka et al, 2010) \\
\hline $\begin{array}{l}\text { Solanum torvum Sw. } \\
\text { [BUBH0000018] }\end{array}$ & Solanaceae & Shrub & W & $\begin{array}{l}\text { khunthai } \\
\text { nara }\end{array}$ & $\mathrm{Fr}$ & Raw/ mixture & 1 & 0.018 & $\begin{array}{l}\text { Yes, (Kamaraj et al. 2011; } \\
\text { Karumari et al. 2014) }\end{array}$ \\
\hline $\begin{array}{l}\text { Lippia alba (Mill.) N.E.Br. ex } \\
\text { Britton \& P.Wilson } \\
\text { [BUBH0000056] }\end{array}$ & Verbenaceae & Shrub & W & onthai bajab & $\mathrm{L}$ & Decoction & 2 & 0.037 & No \\
\hline $\begin{array}{l}\text { Tectona grandis L.f. } \\
\text { [BUBH0000037] }\end{array}$ & Verbenaceae & Tree & $\mathrm{D}$ & $\begin{array}{l}\text { sigun } \\
\text { dongfang }\end{array}$ & $\mathrm{L}$ & Raw/ mixture & 1 & 0.018 & No \\
\hline $\begin{array}{l}\text { Aloe vera (L.) Burm.f. } \\
\text { [BUBH0000022] }\end{array}$ & Xanthorrhoeaceae & Herb & $\mathrm{D}$ & aloe vera & $L, L x$ & Raw/ Mixture & 1 & 0.018 & No \\
\hline $\begin{array}{l}\text { Alpinia nigra (Gaertn.) Burtt } \\
{[\mathrm{BUBH} 0000044]}\end{array}$ & Zingiberaceae & Herb & W & tharai & $\mathrm{Lt}$ & Raw/ mixture & 1 & 0.018 & $\begin{array}{l}\text { Yes, (Roy \& Swargiary } \\
\text { 2009; Roy et al. 2009) }\end{array}$ \\
\hline $\begin{array}{l}\text { Curcuma caesia Roxb. } \\
\text { [BUBH0000008] }\end{array}$ & Zingiberaceae & Herb & $\mathrm{W}$ & haldi gwswm & $\mathrm{T}$ & Raw/ mixture & 2 & 0.037 & No \\
\hline
\end{tabular}

L- leaves, Lt- tender leaves, Fr- fruits, R- roots, WP- whole plant, B- barks, Lx- latex, T- tuber, St- stem, Sd- seed, Fl- flower. Mode of use is oral in all the plant and its traditional formulations, $\mathrm{W}$ - wild, D - domesticated, TF - traditional formulation 
However, from 23 other families, only one species was reported. The present study indicates the richness of plants used against helminth infection by the traditional healers of tribal communities of the Kokrajhar district of Assam. It has also been seen that most traditional healers deal with humans, except a few who also deal with livestock such as cows, goats, etc. The survey report also showed that most (58\%) of the reported plants are wild in habitats that are not domesticated. Figure 3 shows the number of plant citations. The most commonly used anthelmintic plant was $A$. paniculata with 27 citations, followed by $A$. scholaris (14 citations), $A$. comosus (12 citations), and $A$. indica (10 citations). Out of the reported 64 sp., 43 sp. were mentioned once by the informants. In terms of availability, the survey found that most plants are readily available and accessible to people, except few plants. According to traditional healers, plants like A. aspera, P. bakeri, T. trilobatum, L. crustacea, and R. tetraphylla were difficult to get.

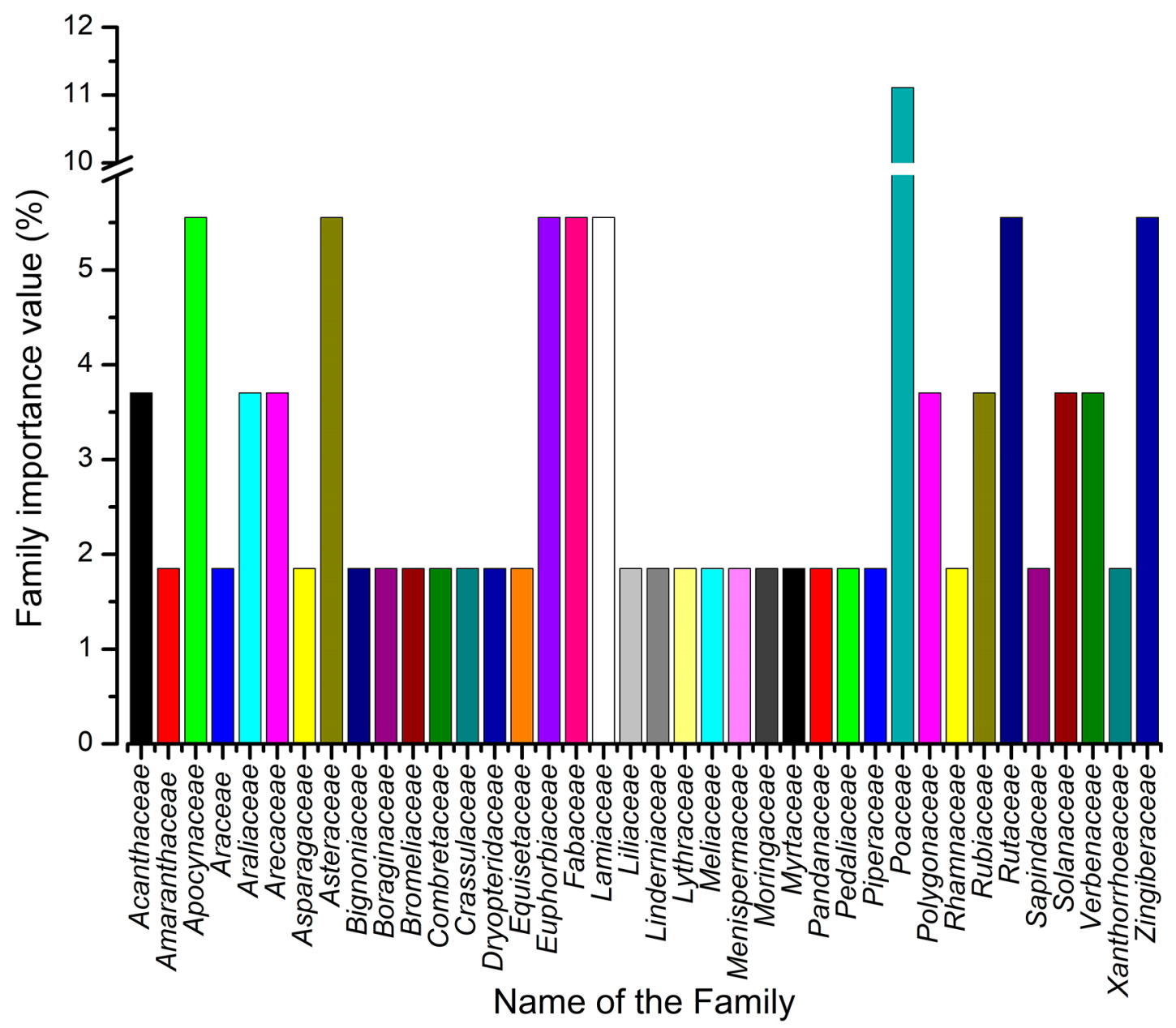

Figure 2. Number of plant families and the relative importance used in the traditional medicinal system by tribal communities against helminthiasis.

Regarding the use of plant parts, leaves were found to be the most commonly used plant parts against helminthiasis. Out of the reported 64 plants, leaves were used in 41 plants to prepare the traditional medicine. Barks, tubers, fruits, roots, and stems were other plant parts commonly used in herbal preparations (Figure 4). In two plant sp. (E. neriifolia and $A$. vera), latex was also used for herbal preparation. Out of 41 leaf-use citations, seven citations showed the use of tender leaves for herbal preparations. In two plants, H. sibthorpioides and $H$. asiatica, the whole aerial part of the plant is used to prepare the ethnomedicine. In nine plants, more than one part is used to prepare the traditional medicines against helminth infection. It is also observed that the TMs are formulated in five different methods: decoction, infusion, raw, infusion and mixture, and raw and mixture (Figure 4). The process of decoction and infusion is the most common method of TM. Most of the plants reported during the survey were found to be eaten as raw material mixed with other plants or ingredients. The present study also observed that most of the traditional medicines were consumed orally. 


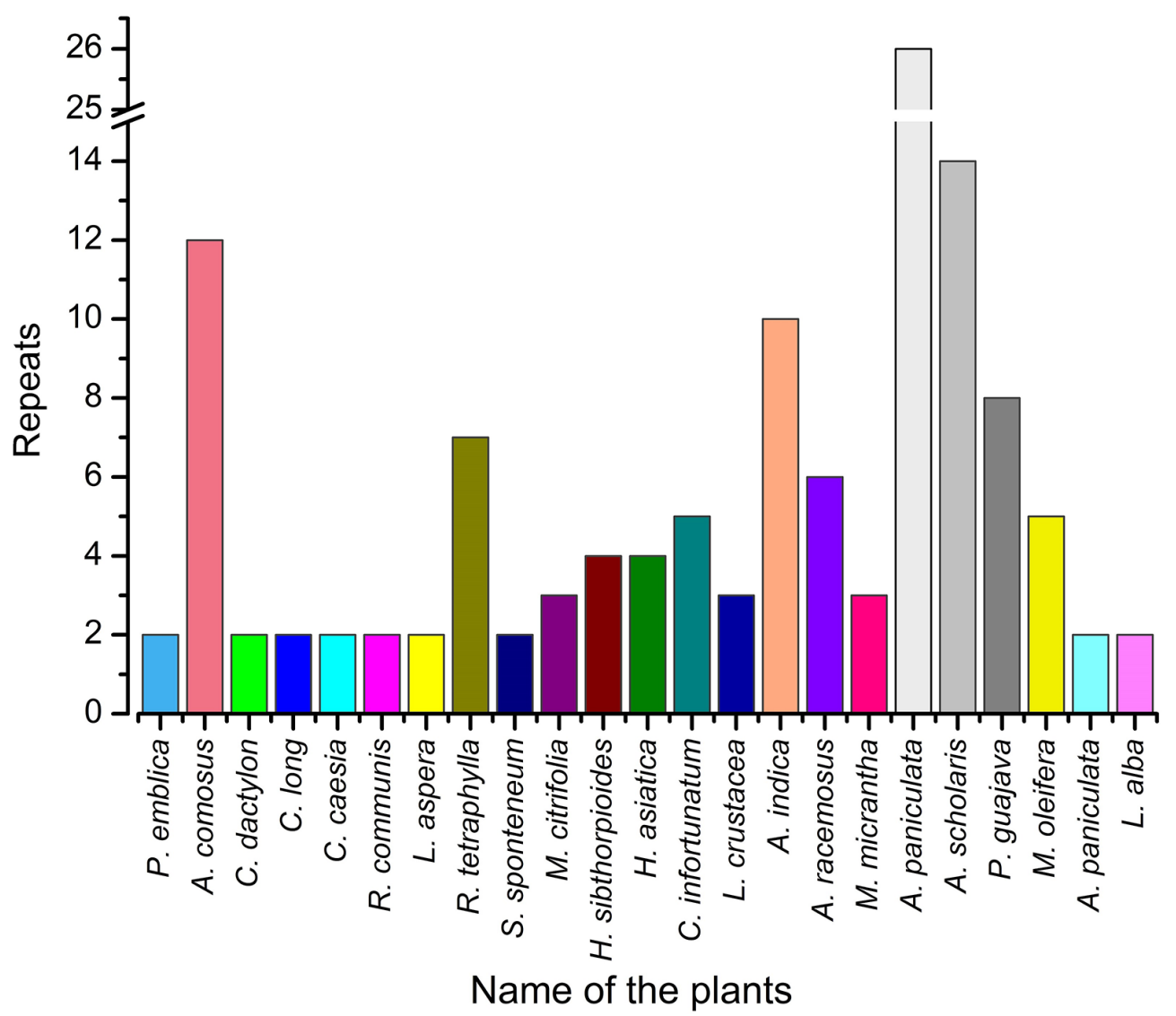

Figure 3. Showing the list of plant citations by village Kaviraja and older people.

\section{Discussion}

Plants are a source of natural medicines since ancient times because of their rich phytochemical contents. For ages, people living in rural areas, especially from third-world countries, use plant-based herbal medicines for treating different ailments. Gifted with rich flora and fauna, India is rich in ethnomedicinal knowledge systems. In the present study, putative anthelmintic plants traditionally used by the Bodo community of Kokrajhar district of India were surveyed and documented. Traditional medicine is an essential component of the health care system of most developing countries. Documentation of indigenous use of medicinal plants is important for several reasons. It helps conservation of traditional heritage and biodiversity and also opens up avenues to search for new drugs for diseases (Boadu \& Asase 2017). Available literature suggests that the traditional wisdom of ethnomedicine is mainly possessed by aged people. People believe that older people possess more ethnomedicinal knowledge and have more understanding than younger people. The present study also observed that about $74 \%$ of the total informants were above 50 years old.

Likewise, many studies also found that the traditional healers are generally above 50 years old (Muthee et al. 2011; Baratti-Mayer et al. 2019). In our earlier studies, we also observed that most of the informants having anthelmintic ethnomedicinal information from three districts Udalguri, Baksa and Udalguri of BTR, were above 50 years old (Swargiary et al. 2019a, b; Swargiary et al. 2020). However, most of the time, traditional healers are poor, uneducated and live in the remotest part of the state. Muthee et al. (2011) observed that most traditional herbalists from the Loitoktok district of Kenya are illiterate. Of the $11 \mathrm{CDBs}$, most of the informants from Kokrajhar and Kochugaon CDBs were literate, while informants from other CDBs were mainly illiterate. Meanwhile, the number of plants cited by literate (4.6 plant citations) and illiterate ( 5.0 plant citations) informants were found to be more or less the same. About $72 \%$ of the total informants were professional healers who practice traditional healing daily and earn little money. In comparison, $28 \%$ were older knowledgeable people who involve neither in practical traditional healing practice nor money earning process. 

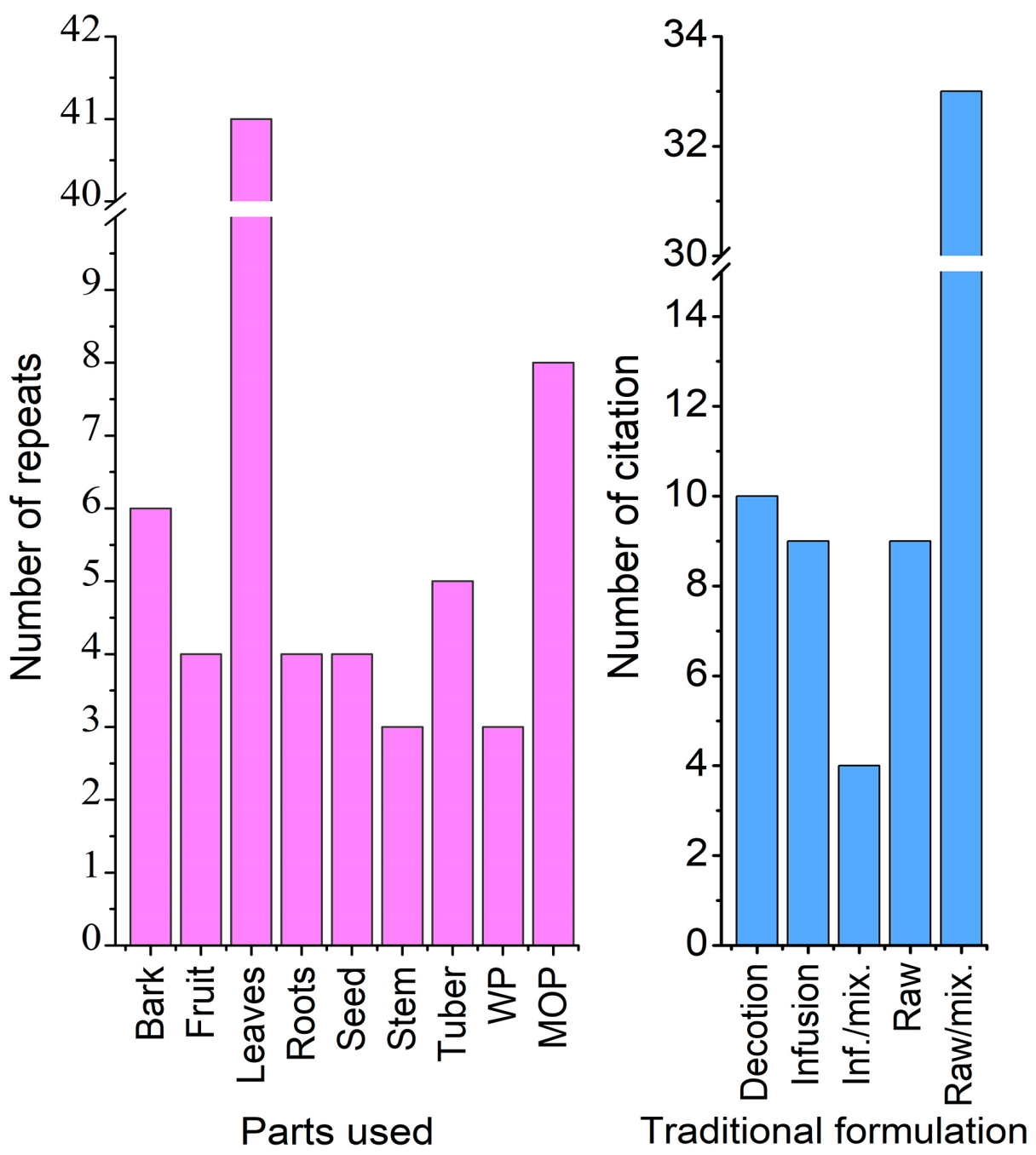

Figure 4. Graph showing (a) the frequency of plant parts used during traditional herbal preparations and (b) types of traditional formulation practices by the tribal communities of Kokrajhar district. WP-whole plant, MOP - more than one part.

The present study shows the richness of plants used in the treatment of helminth infection by the local healers among the Bodo community of the Kokrajhar district. It has also been seen that most traditional healers deal with humans, while a few herbalists also deal with livestock such as cows, goats, etc. Herbal preparations are generally prepared by a mixture of different plants, most commonly, wild in habitat. Our survey report also showed that most (58\%) of the reported plants are wild in habitats that are not domesticated. Wintola \& Afolayan (2015) also surveyed the anthelmintic plants of Amathole district municipality of the Eastern Cape Province of South Africa, showing Poaceae as one of the most popular anthelmintic plant families. Leaves and roots were found to be the most commonly used parts. It has also come to our observation that most traditional healers do not use any precise and standardised methods of herbal formulation. Based on the nature of the infection, doses of the traditional formulations are also altered. However, in most cases, it has been reported that doses are increased or decreased on the basis of the age of the patient. Furthermore, there was no uniformity in the doses among the informants.

The process of decoction and infusion was the most common method of herbal formulation in the present study. Wintola \& Afolayan (2015) also observed similar findings from a study in the Amathole district municipality of the Eastern Cape province, South Africa. Herbal preparations are generally a mixture of several ingredients or plant parts. The present study also observed that more than $56 \%$ of the reported plants are consumed orally as raw mixtures with other plants. Many studies have also reported similar results emphasising boiling or soaking in water and drying and grinding as the most preferred methods of the traditional formulation (Teklehaymanot \& Giday 2007; Nanyingi et al. 2008; Muthee et al. 2011). Our survey also found that all the traditional formulations are served and taken through the oral route. Many such ethnomedicinal survey studies have also found similar results (Ndob 
et al. 2016). The oral route of administration is the most common in all the traditional formulations and plants cited.

Available literature suggests that about $50 \%$ of the total reported plants have scientific validation about their anthelmintic property that are previously investigated by other researchers. The alcoholic extracts of leaves, barks and seeds of $A$. paniculata were investigated by many researchers showing potent anthelmintic activity (Singh et al. 2009; Kamaraj et al. 2011; Padma et al. 2012). Following our survey findings, the anthelmintic activity of $A$. comosus, A. indica, P. guajava, M. oleifera, A. racemosus, and C. infortunatum was reported by (Chagas et al. 2008; Das et al. 2011; Molla \& Bandyopadhyay 2014; Cabardo \& Portugaliza 2017). In a recent study, Alstonia scholaris, Cardiospermum halicacabum, Hydrocotyle sibthorpioides, and Hypericum japonicum were reported to possess substantial in vitro anthelmintic activity (Swargiary et al. 2021). The alcoholic leaves extract of M. citrifolia have scientific studies for their anthelmintic activity (Kumar et al. 2010). The presence of experimental evidence and higher citations of medicinal plants by traditional healers suggest the significance and importance of ethnomedicines.

\section{Conclusions}

The present study revealed that the ethnomedicinal knowledge of helminth infection is widespread among the tribal communities of Assam. Although there is rich ethnomedicinal knowledge, most traditional healers do not follow the precise methodology of herbal preparation. Depending on the severity of the diseases, herbal formulations were prepared. The formulations and doses are prepared in the form of the numbers of leaves, fruits, flowers. Therefore, a detailed bioactivity-based study is needed to develop a precise formulation method and better efficacy. The study reported several plants with no scientific validation. Therefore, further study needs to be carried out to explore the bioactivity of the plants.

\section{Declarations}

Ethics approval and consent to participate: Not applicable.

Consent for publication: All authors read the final manuscript and approved it for publication.

Availability of data and materials: All data related to the manuscript is present within the paper.

Funding: Science and Engineering Research Board, Government of India (File no. EEQ/2017/000071)

Authors' contributions: AS designed the work and wrote the manuscript, MD and MK Roy both involved in collecting ethnomedicinal data.

\section{Acknowledgement}

AS would like to thank SERB, Govt. of India, for providing financial assistance in a research project (Project no. EEQ/2017/000071). Authors are also thankful to the village Kaviraja and older people for providing ethnomedicinal information. Furthermore, we are very much thankful to Dr Sanjib Baruah Asstt. Prof, Department of Botany, for helping in scientific validation of the sample plants.

\section{Literature cited}

Adeniyi A, Asase A, Ekpe PK, Asitoakor BK, Adu-Gyamfi A, Avekor PY. 2018. Ethnobotanical study of medicinal plants from Ghana; confirmation of ethnobotanical uses, and review of biological and toxicological studies on medicinal plants used in Apra Hills Sacred Grove. Journal of Herbal Medicine 14:76-87.

Agarwal S, Jacob S, Chettri N, Bisoyi S, Badarinath DK, Vedamurthy AB, Krishna V, Hoskeri HJ. 2011. Evaluation of in vitro anthelmintic activity of Leucas aspera extracts. Pharmacognosy Journal 3:77-80.

Aggarwal R, Bagai U. 2017. Effect of Punica granatum fruit peel on glucose-6-phosphate dehydrogenase and malate dehydrogenase in amphistome Gastrothylax indicus. Journal of Parasitic Diseases 41:16-20.

Ahmed M, Laing MD, Nsahlai IV. 2014. In vivo effect of selected medicinal plants against gastrointestinal nematodes of sheep. Tropical Animal Health and Production 46:411-417.

Ayaz M, Junaid M, Subhan F, Ullah F, Sadiq A, Ahmad S, Imran M, Kamal Z, Hussain S, Shah SM. 2014. Heavy metals analysis, phytochemical, phytotoxic and anthelmintic investigations of crude methanolic extract, subsequent fractions and crude saponins from Polygonum hydropiper L. BMC Complementary and Alternative Medicine 14:465.

Aziz MA, Khan AH, Adnan M, Ullah H. 2018. Traditional uses of medicinal plants used by indigenous communities for veterinary practices at Bajaur Agency, Pakistan. Journal of Ethnobiology and Ethnomedicine 14:11. 
Baratti-Mayer D, Baba Daou M, Gayet-Ageron A, Jeannot E, Pittet-Cuénod B. 2019. Sociodemographic Characteristics of Traditional Healers and Their Knowledge of Noma: A Descriptive Survey in Three Regions of Mali. International Journal of Environmental Research and Public Health 16(22):4587.

Barkaoui M, Katiri A, Boubaker H, Msanda F. 2017. Ethnobotanical survey of medicinal plants used in the traditional treatment of diabetes in Chtouka Ait Baha and Tiznit (Western Anti-Atlas), Morocco. Journal of Ethnopharmacology 198:338-350.

Bazh EK, El-Bahy NM. 2013. In vitro and in vivo screening of anthelmintic activity of ginger and curcumin on Ascaridia galli. Parasitology Research 112:3679-3686.

Behera DR, Bhatnagar S. 2018. Assessment of macrofilaricidal activity of leaf extracts of Terminalia sp. against bovine filarial parasite Setaria cervi. The Journal of Infection and Public Health 11:643-647.

Boadu AA, Asase A. 2017. Documentation of Herbal Medicines Used for the Treatment and Management of Human Diseases by Some Communities in Southern Ghana. Evidence-Based Complementary and Alternative Medicine 2017:3043061. doi:10.1155/2017/3043061

BTC. 2003. Accord. Memorandum of Settlement on Bodoland Territorial Council. https://peacemaker.un.org/sites/peacemaker.un.org/files/IN_030210_MemorandumSettlementBodolandTerritorial Council.pdf. Retrieved on 28-05-2021.

Cabardo DE, Portugaliza HP. 2017. Anthelmintic activity of Moringa oleifera seed aqueous and ethanolic extracts against Haemonchus contortus eggs and third-stage larvae. International Journal of Veterinary Science and Medicine 5:30-34.

Census of India. 2011. Assam- Series -19 Part-XII-B, District Census Handbook Kokrajhar. Directorate of Census Operations, Assam. Ministry of Home Affairs, Guwahati-07. website: http://www.censusindia.gov.in.

Chagas ACS, Vieira LS, Freitas, AR, Araújo MRA, Araújo-Filho JA, Araguão WR, Navarro AM. 2008. Anthelmintic efficacy of neem (Azadirachta indica A. Juss) and the homeopathic product Fator Vermes in Morada Nova sheep. Veterinary Parasitology 151:68-73.

Choudhury PR, Choudhury MD, Ningthoujam SS, Das D, Nath D, Das Talukdar A. 2015. Ethnomedicinal plants used by traditional healers of North Tripura district, Tripura, North East India. Journal of Ethnopharmacology 66:135-148.

Costa CT, Bevilaqua CM, Maciel MV, Camurça-Vasconcelos AL, Morais SM, Monteiro MV, Farias VM, da Silva MV, Souza MM. 2006. Anthelmintic activity of Azadirachta indica A. Juss against sheep gastrointestinal nematodes. Veterinary Parasitology 137:306-310.

Das JK, Choudhury S, Adhikary SB, Das B, Samanta S, Mandal SC, Dey SP. 2011. Anthelmintic activity of Clerodendrum viscosum. Oriental Pharmacy and Experimental Medicine 11:119-122.

Das M, Laha R. 2017. Parasitic infections of cattle in northeastern region of India - an overview. Archives of Parasitology 1:107.

Das NJ, Saikia SP, Sarkar S, Devi K. 2006. Medicinal plants of North-Kamrup district of Assam used in the primary healthcare system. Indian Journal of Traditional Knowledge 5:489-493.

Das S, Akhter R, Huque S, Anwar R, Das P, Tanni KA, Shahriar M. 2017. In vitro anthelmintic activity of leaf extracts of four different types of Calamus species. Pharmacy \& Pharmacology International Journal 5:72-76.

Deori K, Yadav AK. 2016. Anthelmintic effects of Oroxylum indicum stem bark extract on juvenile and adult stages of Hymenolepis diminuta (Cestoda), an in vitro and in vivo study. Parasitology Research 115:1275-1285.

Dixit AK, Das G, Singh Baghel RP. 2017. Gastrointestinal helminthosis: prevalence and associated determinants in goats of Jabalpur, India. Journal of Parasitic Diseases 41:414-416.

Domingues LF, Giglioti R, Feitosa KA, Fantatto RR, Rabelo MD, et al. 2013. In-vitro and in-vivo evaluation of the activity of pineapple (Ananas comosus) on Haemonchus contortus in Santa Ines sheep. Veterinary Parasitology 197:263-270.

Dwivedi S, Dwivedi A, Kapadia R, Kaul S. 2008. Anthelmintic activity of alcoholic and aqueous extract of fruits of Terminalia chebula Retz. Ethnobotanical Leaflets 12:741-743. 
Gainza YA, Dominguis LF, Parez OP, Rabelo MD, Lopez ER, Chagas AC. 2015. Anthelmintic activity in vitro of Citrus sinensis and Melaleuca quinquenervia essential oil from Cuba on Haemonchus contortus. Industrial Crops and Products 76:647-652.

Gogtay NJ, Bhatt HA, Dalvi SS, Kshirsagar NA. 2002. The use and safety of non-allopathic Indian medicines. Drug Safety 25(14):1005-1019.

Gollen B, Mehla J, Gupta P. 2018. Clitoria ternatea Linn.: a herb with potential pharmacological activities: Future prospects as therapeutic Herbal Medicine. Journal of Pharmacological Reports 3:141.

Gupta S, Baweja S, Dubey R, Singh D, Singh S. 2012. Combinational effect of Asparagus racemosus, Andrographis paniculata and Discorea villosa for anthelmintic and antimicrobial potential. International Journal of Pharmaceutical Sciences Review and Research 16:104-107.

Hordegen P, Cabaret J, Hertzberg H, Langhans W, Maurer V. 2006. In vitro screening of six anthelmintic plant products against larval Haemonchus contortus with a modified methyl-thiazolyl-tetrazolium reduction assay. Journal of Ethnopharmacology 108:85-89.

Hussain W, Ullah M, Dastagir G, Badshah L. 2018. Quantitative ethnobotanical appraisal of medicinal plants used by inhabitants of lower Kurram, Kurram agency, Pakistan. Avicenna Journal of Phytomedicine 8:313-329.

Iqbal Z, Lateef M, Jabbar A, Gilani AH. 2010. In vivo anthelmintic activity of Azadirachta indica A. Juss seeds against gastrointestinal nematodes of sheep. Veterinary Parasitology 168:342-345.

Kalarickal DC, Samraj S, Udayan D, Narayanan PM, Ramachandran S, Gouri SS. 2015. Effect of various extracts of Ocimum sanctum and Mallotus phillipensis on Setaria digitata. Pharmacognosy Journal 7:344-347.

Kamaraj C, Rahuman AA, Elango G, Bagavan A, Abduz Zahir J. 2011. Anthelmintic activity of botanical extracts against sheep gastrointestinal nematodes, Haemonchus contortus. Parasitology Research 109:37-45.

Kanojiya D, Shanker D, Sudan V, Jaiswal AK, Parashar R. 2015. Anthelmintic activity of Ocimum sanctum leaf extract against ovine gastrointestinal nematodes in India. Research in Veterinary Science 99:165-170.

Karumari RJ, Sumathi S, Vijayalakshmi K, Balasubramanian SE. 2014. Anthelmintic efficacy of Sesbania grandiflora leaves and Solanum torvum fruits against the nematode parasite Ascaridia galli. American Journal of Ethnomedicine 1:326-333.

Khadatkar SN, Manwar J, Bhajipale NS. 2008. In vitro anthelmintic activity of root of Clitoria ternatea Linn. Pharmacognosy Magazine 4:148-150.

Kone WM, Vargas M, Keiser J. 2012. Anthelmintic activity of medicinal plants used in Côte d'Ivoire for treating parasitic diseases. Parasitology Research 110:2351-2362.

Kotoky J, Das PN. 2008. Medicinal plants used for liver diseases in some parts of Kamrup district of Assam, a North Eastern State of India. Fitoterapia 79:384-387.

Kozan E, Kupeli E, Yesilada E. 2006. Evaluation of some plants used in Turkish folk medicine against parasitic infections for their in vivo anthelmintic activity. Journal of Ethnopharmacology 108:211-216.

Kumar KP, Panda DS, Nanda UN, Khuntia S. 2010. Evaluation of antibacterial, antifungal and anthelmintic activity of Morinda citrifolia L. (Noni). International Journal of PharmTech Research 2:1030-1032.

Kumar P, Nain MS. 2013. Agriculture in India: A SWOT analysis. Indian Journal of Applied Research 3:3-6.

Lobo DA, Velayudhan R, Chatterjee P, Kohli H, Hotez PJ. 2011. The neglected tropical diseases of India and South Asia: review of their prevalence, distribution, and control or elimination. PLOS Neglected Tropical Diseases 5:e1222.

Lunkad AS, Agrawal MR, Kothawade SN. 2016. Anthelmintic activity of Bryophyllum pinnatum. Research Journal of Pharmacognosy and Phytochemistry 8:21-24.

Mahadev ND, Thorat, AT, Vitthal BP. 2017. An evaluation of anthelmintic activity of Ricinus communis Linn. leaves by using different type of solvent. Journal of Pharmacognosy and Phytochemistry 6:1845-1847. 
Maphosa V, Masika PJ. 2010. Ethnoveterinary uses of medicinal plants: a survey of plants used in the ethnoveterinary control of gastro-intestinal parasites of goats in the Eastern Cape Province, South Africa. Pharmaceutical Biology 48:697-702.

Meenakshisundaram A, Harikrishnan TJ, Anna T. 2016. Anthelmintic activity of Sesbania grandiflora against gastrointestinal nematodes of sheep. Indian Journal of Animal Sciences 86:143-148.

Mishra D, Sarkar DK, Nayak BS, Rout PK, Ellaiah P, Ramakrishna S. 2010. Phytochemical investigation and evaluation of anthelmintic activity of extract from leaves of Eupatorium odoratum linn. Indian Journal of Pharmaceutical Education and Research 44:369-374.

Mittermeier RA, Turner WR, Larsen FW, Brooks TM, Gascon C. 2011. Global biodiversity conservation: the critical role of hotspots, in: F.E. Zachos, J.C. Habel (Eds.), Biodiversity Hotspots, Springer Publishers, London, pp. 3-22.

Molla SH, Bandyopadhyay PK. 2014. In-vitro anthelmintic activity of Psidium guajava against sheep gastrointestinal nematode, Haemonchus contortus. Environment and Ecology 32:616-621.

Molla SH, Bandyopadhyay PK. 2016. In vitro and in vivo anthelmintic activity of Murraya koenigii against gastrointestinal nematodes of sheep. Journal of Parasitic Diseases 40:362-368.

Mostafa Kamal ATM, Ahmed Chowdhury KA, Hossen Chy MM, Shill LK, Chowdhury S, Hossen Chy MA, Zia Habib M. 2015. Evaluation of anthelmintic activity of seeds of Sesamum indicum $L$. and fruits of Capsicum frutescens $L$. Journal of Pharmacognosy and Phytochemistry 3:256-259.

Moyeenul Huq AKM, Jamal JA, Stanslas J. 2014. Ethnobotanical, phytochemical, pharmacological, and toxicological aspects of Persicaria hydropiper (L.) Delarbre. Evidence-Based Complementary and Alternative Medicine 2014:782830.

Muthee JK, Gakuya DW, Mbaria JM, Kareru PG, Mulei CM, Njonge FK. 2011. Ethnobotanical study of anthelmintic and other medicinal plants traditionally used in Loitoktok district of Kenya. Journal of Ethnopharmacology 135:1521.

Nanyingi MO, Mbaria JM, Lanyasunya AL, Wagate CG, Koros KB, Kaburia HF, Munenge RW, Ogara WO. 2008. Ethnopharmacological survey of Samburu district, Kenya. Journal of Ethnobiology and Ethnomedicine 4:14.

Narzary H, Brahma S, Basumatary S. 2013. Wild edible vegetables consumed by Bodo tribe of Kokrajhar district (Assam), North East India. Archives of Applied Science Research 5:182-190.

Nasai NB, Abba Y, Abdullah FF, Marimuthu M, Tijjani A, Sadiq MA, Mohammed K, Chung EL, Omar MA. 2016. In vitro larvicidal effects of ethanolic extract of Curcuma longa Linn. on Haemonchus larval stage. Veterinary World 9:417420.

Ndhlala AR, Ghebrehiwot HM, Ncube B, Aremu AO, Gruz J, Subrtová M, Dolezal K, du Plooy CP, Abdelgadir HA, Van Staden J. 2015. Antimicrobial, anthelmintic, activities and characterisation of functional phenolic acids of Achyranthes aspera Linn.: A medicinal plant used for the treatment of wounds and ringworm in East Africa. Frontiers of Pharmacology 6:274.

Ndob IB, Mengome LE, Bourobou HB, Banfora YL, Bivigouab F. 2016. Ethnobotanical survey of medicinal plants used as anthelmintic remedies in Gabon. Journal of Ethnopharmacology 191:360-371.

Nilani P, Kumar PM, Duraisamy B, Dhamodaran P, Jeyaprakash MR. 2012. Anthelmintic activity of Moringa Oleifera seed oil - validation of traditional use. Journal of Advanced Scientific Research 3:65-66.

Padma Y, Devi N, Manjunatha B, Philip GH, Venkata Raju RR. 2012. Preliminary phytochemical screening and anthelmintic activity of Andrographis echioides Nees. Journal of Pharmaceutical Research 5:4801-4803.

Pal D, Pandab K. 2010. Evaluation of anthelmintic activities of aerial parts of Cynodon dactylon Pers. Ancient Science of Life 30:12-13.

Pendry B, Busia K, Bell CM. 2005. Phytochemical evaluation of selected antioxidant containing medicinal plants for use in the preparation of an herbal formula-a preliminary study. Chemistry \& Biodiversity 2:917-922. 
Pina-Vázquez DM, Mayoral-Pena Gómez-Sanchez M, Salazar-Olivo LA, Arellano-Carbajal. 2017. Anthelmintic effect of Psidium guajava and Tagetes erecta on wild-type and levamisole-resistant Caenorhabditis elegans strains. Journal of Ethnopharmacology 202:92-96.

Purkayastha J, Nath SC, Islam M. 2005. Ethnobotany of medicinal plants from Dibru-Saikhowa Biosphere Reserve of Northeast India. Fitoterapia 76:121-127.

Rajarajan AT, Vijayasree VG, Kenichi W, Vijaya Kumar S, Narasimman G, Sadish kumar S. 2009. Anthelmintic and antimicrobial properties of peels of Citrus sinensis. Pharmacologyonline 1:363-368.

Rajeshwar Y, Lalitha R. 2013. Preliminary phytochemical screening and in vitro anthelmintic effects of Acmella paniculata plant extracts. Biolife 1:106-112.

Roy B, Dasgupta S, Tandon V. 2009. Ultrastructural observations on Fasciolopsis buski and its alterations caused by shoot extract of Alpinia nigra. Microscopy Research and Technique 72:61-66.

Roy B, Swargiary A. 2009. Anthelmintic efficacy of ethanolic shoot extract of Alpinia nigra on tegumental enzymes of Fasciolopsis buski, a giant intestinal parasite. Journal of Parasitic Diseases 33:48-53.

Salam N, Azam S. 2017. Prevalence and distribution of soil-transmitted helminth infections in India. BMC Public Health 17:201.

Samy RP, Ignacimuthu S. 2000. Antibacterial activity of some folklore medicinal plants used by tribals in Western Ghats of India. Journal of Ethnopharmacology 69:63-71.

Shankar R, Lavekar GS, Deb S, Sharma BK. 2012. Traditional healing practice and folk medicines used by Mishing community of North East India. Journal of Ayurveda and Integrative Medicine 3:124-129.

Sheela S, Viswanathan PK. 2009. Anthelmintic activity of leaves of Chromolaena odorata (Linn) King \& Robinson Journal of Pharmaceutical Research 2:868-869.

Singh S, Mehta A, John J, Mehta P. 2009. Anthelmintic Potential of Andrographis paniculata, Cajanus cajan and Silybum marianum. Pharmacognosy Journal 1:243-245.

Sonowal R, Barua I. 2017. Ethnomedical Practices among the Tai-Khamyangs of Assam, India. Studies on EthnoMedicine 5:41-50.

Swargiary A, Boro H, Brahma BK, Rahman S. 2013. Ethno-botanical study of antidiabetic medicinal plants used by the local people of Kokrajhar District of Bodoland Territorial Council, India. Journal of Medicinal Plants Studies 1:5158.

Swargiary A, Daimari A, Daimari M, Basumatary N, Narzary E. 2016. Phytochemicals, antioxidant, and anthelmintic activity of selected traditional wild edible plants of lower Assam. Indian Journal of Pharmacology 48:418-423.

Swargiary A, Daimari M, Roy M K. 2020. Survey and documentation of anthelmintic plants used in traditional medicine system of tribal communities of Udalguri district of Assam, India. Journal of Applied Pharmaceutical Science 10(1):46-54.

Swargiary A, Roy M K, Daimari M. 2019a. Survey and documentation of ethnobotanicals used in the traditional medicines system of tribal communities of Chirang district of assam against helminthiasis. Biomedical and Pharmacology Journal 12(4).

Swargiary A, Roy M K, Daimari M. 2019b. Survey and documentation of putative anthelmintic plants used in ethnomedicinal systems of tribal communities of Baksa District of Assam. Medicinal Plants 11(4):368-379.

Swargiary A, Roy MK, Verma AK. 2021. In vitro study of the antioxidant, antiproliferative, and anthelmintic properties of some medicinal plants of Kokrajhar district, India. Journal of Parasitic Diseases 2021. https://doi.org/10.1007/s12639-021-01410-0.

Tamuli P, Ghosal A. 2017. Ethnomedicinal plants used by major ethnic groups of Assam (India) for curing skin diseases. International Journal of Herbal Medicine 5:140-144.

Tandon V, Yadav AK, Roy B, Das B. 2011. Phytochemicals as cure of worm infections in traditional medicine systems, in: Srivastava U.C. and Kumar S. (Edn.), Emerging Trends in Zoology, Narendra Publishing House, New Delhi, pp. 351-378. 
Tardio J, Pardo-de Santayana M. 2008. Cultural importance indices: a comparative analysis based on the useful wild plants of Southern Cantabria (northern Spain). Economic Botany 62:24-39.

Teklehaymanot T, Giday M. 2007. Ethnobotanical study of medicinal plants used by the people of Zegie Peninsula, Northwestern Ethiopia. Journal of Ethnobiology and Ethnomedicine 3:12.

Urban J, Kokoska L, Langrova I, Matejkova J. 2008. In vitro anthelmintic effects of medicinal plants used in Czech Republic. Pharmaceutical Biology 46:808-813.

Verpoorte R, Choi Y, Kim H. 2005. Ethnopharmacology and systems biology: a perfect holistic match. Journal of Ethnopharmacology 100:53-56.

Vinayaka KS, Nandini KC, Rakshitha MN, Ramya M, Shruthi J, Shruthi VH, Prashith Kekuda TR, Raghavendra H.2010. Proximate composition, antibacterial and anthelmintic activity of Capsicum frutescens (L.) Var. Longa (Solanaceae) Leaves. Pharmacognosy Journal 2:486-491.

Vitalini S, Iriti M, Puricelli C, Ciuchi D, Segale A, Fico G. 2013. Traditional knowledge on medicinal and food plants used in Val San Giacomo (Sondrio, Italy) - an alpine ethnobotanical study. Journal of Ethnopharmacology 145:517529.

Watkins F, Pendry B, Corcoran O, Sanchez-Medina A. 2011. Anglo-Saxon pharmacopoeia revisited: a potential treasure in drug discovery. Drug Discovery Today 16(23):1069-1075.

WHO. 2013. WHO Traditional Medicine Strategy 2014-23. https://www.who.int/publications/i/item/ 9789241506096

Wintola OA, Afolayan AJ. 2015. An inventory of indigenous plants used as anthelmintics in Amathole district municipality of the Eastern Cape province, South Africa. African Journal of Traditional, Complementary and Alternative Medicine 12:112-121.

Yadav AK, Nath P. 2017. Anthelmintic effects and toxicity of Cynodon dactylon (L.) Pers. in rodent models. Journal of Intercultural Ethnopharmacology 6:407-413.

Yones DA, Badary DM, Sayed HM, Bayoumi SA, Khalifa AA, El-Moghazy AM. 2016. Comparative evaluation of anthelmintic activity of edible and ornamental pomegranate ethanolic extracts against Schistosoma mansoni. BioMed Research International 2016:2872708.

Zahir AA, Rahuman AA, Bagavan A, Geetha K, Kamaraj C, Elango G. 2012. Evaluation of medicinal plant extracts and isolated compound epicatechin from Ricinus communis against Paramphistomum cervi. Parasitology Research 111:1629. 